\title{
Pruning trees, complexity cost may not be a good approach: initial evidence
}

\author{
Cristiano Mauro Assis Gomes (LAICO, Universidade Federal de Minas Gerais) \\ Heitor Blesa Farias (LAICO, Universidade Federal de Minas Gerais) \\ Jhonys de Araujo (LAICO, Universidade Federal de Minas Gerais) \\ Enio Galinkin Jelihovschi (LAICO, Universidade Estadual de Santa Cruz)
}

\section{Background}

The Laboratory for Cognitive Architecture Mapping [Laboratório de Investigação da Arquitetura Cognitiva (LAICO)] was founded in 2009 in the department of psychology of the Federal University of Minas Gerais, Brazil. Its mission consists in articulating the psychometrics fields in educational issues and investigating the validity of psychological constructs, particularly related to the educational psychology field. In other words, LAICO aims to contribute to three research lines.

The first involves the construction and validation of psychological tests, particularly related to educational outcomes (Costa, Gomes \& Fleith, 2017; Gomes, \& Golino, 2012; Golino \& Gomes, 2012, 2014b; Golino, Gomes, \& Andrade, 2014; Gomes, 2010b, 2011b, 2021a, 2012b; Gomes \& Borges, 2007, 2008a, 2008c, 2009b, 2009c; Gomes \& Gjikuria, 2017, 2018; Gomes \& Golino, 2012a, 2012b; Golino \& Gomes, 2011; Gomes \& Golino, 2014; Gomes, Golino \& Menezes, 2014; Gomes, 2010c, 2011a, 2013; Gomes, Araujo, \& Jelihovschi, 2020; Gomes \& Golino, 2012c; Gomes et al., 2019; Gomes, Golino, Pinheiro, Miranda, \& Soares, 2011; Gomes \& Linhares, 2018; Gomes et al., 2021; Gomes \& Marques, 2016; Gomes, Marques, \& Golino, 2014; Pinheiro, Gomes, \& Braga, 2009; Rodrigues \& Gomes, 2020; Silveira \& Gomes, 2014; Silveira, Gomes, \& Golino, 2012).

Many of these tests are available for research and teaching in researchgate (Gomes \& Nascimento, 2021a, 2021b, 2021c, 2021d, 2021e, 2021f, 2021g, 2021h, 2021i, 2021j, 2021k, 20211, 2021m, 2021n, 2021o, Gomes, Nascimento \& Araujo, 2021a, 2021b, 2021c, 2021d) 
The second contribution of LAICO involves cooperation with many research centers, concerning the validation of theoretical models or instruments of measurement (Alves, Gomes, Martins, \& Almeida, 2016, 2017, 2018; Araujo, Gomes, Almeida, \& Núñez, 2018; Casanova, Gomes, Bernardo, Núñez, \& Almeida, 2021; Fleith et. al 2020; Fleith \& Gomes, 2019; Fleith, Gomes, Marinho-Araujo, \& Almeida, 2020; Matos, Brown, Gomes, 2019; Monteiro, Almeida, Gomes, \& Sinval, 2020; Golino, Gomes, Commons, Miller, 2014; Golino \& Gomes, 2015, 2019; Martins, Gomes, Alves, \& Almeida, 2018; Andre, Gomes, \& Loureiro, 2016, 2017, 2018, 2020a, 2020b, 2020c; Rosário, Gomes, \& Loureiro, 2019; Sampaio, Loureiro \& Gomes, 2015; Costa, Gomes, Andrade, \& Samulski, 2012; Moura, Gomes, Blanc, Mesquita, \& Ferreira, 2014; Rosa et al., 2013; Cardoso, Seabra, Gomes, \& Fonseca, 2019; Dias et al., 2015; Mecca et al., 2015; Pazeto, Dias, Gomes, \& Seabra, 2019, 2020; Reppold et al., 2015; Gomes \& Borges, 2007, 2008c; Laros, Valentini, Gomes, \& Andrade, 2014; Muniz, Pasian, \& Gomes, 2016; Valentini et al., 2015).

The third contribution of LAICO involves both the applications of innovative statistics and algorithms of machine learning, as well as, the elaboration of original methodologies (Cardoso, Seabra, Gomes, \& Fonseca, 2019; Ferreira \& Gomes, 2017; Fleith \& Gomes, 2019; Gauer, Gomes, \& Haase, 2010; Golino, Andrade, \& Gomes, 2014; Golino \& Gomes, 2012, 2014a, 2014c, 2016, 2019; Golino, Gomes, Amantes, \& Coelho, 2015; Gomes, 2007a, 2007b, 2010a, 2020a, 2020b; Gomes \& Almeida, 2017; Gomes, Almeida, \& Núñez, 2017; Gomes, Amantes, \& Jelihovschi, 2020; Gomes, Araujo, Ferreira, \& Golino, 2014; Gomes, Araujo, Nascimento, \& Jelihovisch, 2018; Gomes \& Borges, 2008b, 2009a; Gomes, Fleith, Marinho-Araujo, \& Rabelo, 2020; Gomes \& Gjikuria, 2017; Gomes \& Golino, 2015; Gomes, Golino, \& Costa, 2013; Gomes, Golino \& Peres, 2016, 2018, 2020; Gomes, Golino, Santos, \& Ferreira, 2014; Gomes \& Jelihovschi, 2016, 2019; Gomes, Lemos, \& Jelihovschi, 2020; Gomes, Linhares, Jelihovschi, \& Rodrigues, 2021; Gomes, Nascimento, \& Peres, 2019; Gomes \& Valentini, 2019; Jelihovschi, \& Gomes, 2019; Matos, Brown, Gomes, 2019; Pereira, Golino \& Gomes, 2019; Pires \& Gomes, 2017, 2018; Ricci, Gomes, Nico, \& Seabra, 2020; Rodrigues \& Gomes, 2020).

\section{Question of Research}

The researchers of LAICO have been using the CART algorithm for tree analysis in many educational and psychological data and many papers resulted from this work, as Gomes and Almeida (2017) and Gomes and Jelihovschi (2019), which discussed the use of decision and regression tree methods in educational datasets. The CART algorithm along with other algorithms for tree analysis require pruning the tree, in order to eliminate the leaves resulting from noise and do not contribute to the prediction of the outcome. The mainstream approach consists in using the cross-validation K-folder and the identification of the cutoff point value which minimizes the error of prediction to do the pruning. This approach is called complexity cost criterion. Another criterion also largely used is the interpretability, which involves pruning the tree by selecting only a number of leaves which are small enough to allow the researcher to interpret them and get a clear view of 
their meaning. The complexity cost criterion is considered an objective criterion, because it uses the cut-point where the tree is pruned to the smallest error of prediction. Compared to it, the interpretability criterion seems to be an arbitrary cutoff point chosen by convenience. However, this apparent adequacy of the complexity cost criterion is a mistake. Two simulation analyses conducted by the researchers of LAICO show that this criterion is not suitable and the interpretability criterion is much more appropriate to analyse a tree.

\section{Scopus of the Paper}

We created a true tree with 20 leaves and ran two simulation analyses (the code is in appendix), the first generating 100 samples of $\mathrm{N}=239$ and the second generating 100 samples of $\mathrm{N}=10000$. We used those simulations to test the adequacy of the complexity cost criterion in pruning the trees, that is, whether by using this criterion the trees were pruned to the correct number of leaves.

\section{Results}

The simulation of $\mathrm{N}=239$ produced pruned trees with an average of 12.21 ( $\mathrm{SD}=3.86$ ) leaves, and a range of 2 to 20 leaves. The simulation of $\mathrm{N}=10000$ produced an average of $53.62(\mathrm{SD}=$ 12.11) leaves for the pruned trees, and a range of 36 to 85 leaves.

The complexity cost criterion generated pruned trees which clearly underestimated the true number of leaves in the small samples $(\mathrm{N}=239)$ and largely overestimated the correct number of leaves in the large samples $(\mathrm{N}=10000$. In short, these results show that the complexity cost criterion does not lead to the correct number of leaves.

\section{Conclusion}

Our study brings initial evidence that the complexity cost criterion may be ineffective. In small samples, there is a tendency to underestimate the correct number of leaves, while in large samples there is a tendency to overestimate the correct number of leaves. Although the interpretability criterion appears to be guided by convenience, it nonetheless may be better than the complexity cost criterion to estimate the correct number of leaves. The lack of objectivity of the pruning guided by this criterion, can be evaluated through an old approach used in the scientific field, that is, the replicability in different samples. The number of leaves and the adequacy of the tree produced by the interpretability criterion can be evaluated by observing the number of times that these leaves occur in different samples in a similar context of study. If the same variables are used in a similar predictive model in one hundred samples, for example, then the leaves that are continually present 
and show consistent and similar values may be seen as valid. Instead of searching a true tree through a criterion to cut the tree, our study suggests that it may be more effective to evaluate the validity of the tree through this old and powerful strategy of the scientific field.

\section{References}

Alves, A. F., Gomes, C. M. A., Martins, A., \& Almeida, L. S. (2018). The structure of intelligence in childhood: age and socio-familiar impact on cognitive differentiation. Psychological Reports, 121(1), 79-92. doi: 10.1177/0033294117723019

Alves, A. F., Gomes, C. M. A., Martins, A., \& Almeida, L. S. (2016). Social and cultural contexts change but intelligence persists as incisive to explain children's academic achievement. PONTE: International Scientific Researches Journal, 72(9), 70-89. doi: 10.21506/j.ponte.2016.9.6

Alves, A. F., Gomes, C. M. A., Martins, A., \& Almeida, L. S. (2017). Cognitive performance and academic achievement: How do family and school converge? European Journal of Education and Psychology, 10(2), 49-56. doi: 10.1016/j.ejeps.2017.07.001

Alves, F. A., Flores, R. P., Gomes, C. M. A., Golino, H. F. (2012). Preditores do rendimento escolar: inteligência geral e crenças sobre ensino-aprendizagem. Revista E-PSI, 1, 97117. https://revistaepsi.com/artigo/2012-ano2-volume1-artigo5/

André, A. M., Gomes, C. M. A., Loureiro, C. M. V. (2016). Escalas Nordoff Robbins: uma revisão bibliográfica. Percepta, 3(2), 117-131, 2016. doi: 10.34018/2318-891X.3(2)117131 André, A. M., Gomes, C. M. A., Loureiro, C. M. V. (2017). Equivalência de itens, semântica e operacional da versão brasileira da Escala Nordoff Robbins de Comunicabilidade Musical. OPUS, 23(2), 153. doi:10.20504/opus2017b2309.

André, A. M. B., Gomes, C. M. A., \& Loureiro, C. M. V. (2018). Reliability Inter-examiners of The Nordoff Robbins Musical Communicativeness Scale Brazilian Version. 11th International Conference of Students of Systematic Musicology, 101-105. http://musica.ufmg.br/sysmus2018/wp-content/uploads/2018/07/Reliability-Interexaminers-of-the-Nordoff-Robbins-Musical-Communicativeness-Scale-BrazilianVersion.pdf

André, A. M. B., Gomes, C. M. A., \& Loureiro, C. M. V. (2020a). Confiabilidade Interexaminadores da Escala de Relação Criança-Terapeuta na Experiência Musical Coativa para validação no contexto brasileiro. Hodie, 20, e64243, 1-18. doi:10.5216/mh.v20.64243

André, A. M. B., Gomes, C. M. A., \& Loureiro, C. M. V. (2020b). Confiabilidade Interexaminadores da versão brasileira da Escala Nordoff Robbins de Comunicabilidade Musical. In Estudos Latino-americanos em Música. vol.2 (pp. 152-163). Artemis. doi:10.37572/EdArt_13210092015 André, A. M. B., Gomes, C. M. A., \& Loureiro, C. M. V. (2020c). Equivalência de itens, semântica e operacional da "Escala de 
Musicabilidade: Formas de Atividade, Estágios e Qualidades de Engajamento." Orfeu, 5(2), 1-22. doi:10.5965/2525530405022020e0010

Araújo, A. M., Gomes, C. M. A., Almeida, L. S., \& Núñez, J. C. (2018). A latent profile analysis of first-year university students' academic expectations. Anales De Psicología / Annals of Psychology, 35(1), 58-67. doi:10.6018/analesps.35.1.299351

Cardoso, C. O., Seabra, A. G., Gomes, C. M. A., \& Fonseca, R. P. (2019). Program for the neuropsychological stimulation of cognition in students: impact, effectiveness, and transfer effect on student cognitive performance. Frontiers in Psychology, 10, 1-16. doi: 10.3389/fpsyg.2019.01784

Casanova, J. R., Gomes, C. M. A., Bernardo, A. B., Núñez, J. C., \& Almeida, L. S. (2021). Dimensionality and reliability of a screening instrument for students at-risk of dropping out from higher education. Studies in Educational Evaluation, 68, 100957. doi: 10.1016/j.stueduc.2020.100957

Costa, V. T., Gomes, C. M. A., Andrade, A. G. P., \& Samulski, Di. M. (2012). Validação das propriedades psicométricas do RESTQ-Coach na versão brasileira. Motriz: Revista de Educação Física, 18(2), 218-232. doi:10.1590/S1980-65742012000200002

Costa, B. C. G., Gomes, C. M. A., \& Fleith, D. S. (2017). Validade da Escala de Cognições Acadêmicas Autorreferentes: autoconceito, autoeficácia, autoestima e valor. Avaliação Psicológica, 16(1), 87-97. doi: 10.15689/ap.2017.1601.10

Dias, N. M., Gomes, C. M. A., Reppold, C. T., Fioravanti-Bastos, A., C., M., Pires, E. U., Carreiro, L. R. R., \& Seabra, A. G. (2015). Investigação da estrutura e composição das funções executivas: análise de modelos teóricos. Psicologia: teoria e prática, 17(2), 140-152. doi: 10.15348/1980-6906/psicologia.v17n2p140-152

Ferreira, M. G., \& Gomes, C. M. A. (2017). Intraindividual analysis of the Zarit Burden Interview: a Brazilian case study. Alzheimers \& Dementia, 13, P1163-P1164. doi: 0.1016/j.jalz.2017.06.1710

Fleith, D. S., Almeida, L. S., Marinho-Araujo, C. M., Gomes, C. M. A., Bisinoto, C., \& Rabelo, M. L. (2020). Validity evidence of a scale on academic expectations for higher education. Paidéia, 30, e3010. doi: 10.1590/1982-4327e3010

Fleith, D. S., \& Gomes, C. M. A. (2019). Students' assessment of teaching practices for creativity in graduate programs. Avaliação Psicológica, 18(3), 306-315. doi: 10.15689/ap.2019.1803.15579.10

Fleith, D, S., Gomes, C. M. A., Marinho-Araujo, C. M., \& Almeida, L. S. (2020). Expectativas de sucesso profissional de ingressantes na educação superior: estudo comparativo. Avaliação Psicológica, 19(3), 223-231. doi: 10.15689/ap.2020.1903.17412.01 
Gauer, G., Gomes, C. M. A., \& Haase V. G. (2010). Neuropsicometria: Modelo clássico e análise de Rasch. In Avaliação Neuropsicológica, (pp. 22-30). Porto Alegre: Artmed, 2010.

Golino, H. F., \& Gomes, C. M. A. (2011). Preliminary internal validity evidences of two Brazilian Metacognitive Tests. International Journal of Testing, 26, 11-12. https://www.intestcom.org/files/ti26.pdf

Golino, H. F., \& Gomes, C. M. A. (2012). The Structural validity of the Inductive Reasoning Developmental Test for the measurement of developmental stages. International Journal of Testing, 27, 10-11.

https://www.researchgate.net/publication/269985854_The_Structural_Validity_of_the_I nductive_Reasoning_Developmental_Test_for_the_Measurement_of_Developmental_S tages

Golino, H. F., \& Gomes, C. M. A. (2014a). Four Machine Learning methods to predict academic achievement of college students: a comparison study. Revista E-Psi, 1, 68101. Retirado de https://revistaepsi.com/artigo/2014-ano4-volume1-artigo4/

Golino, H.F., \& Gomes, C.M.A. (2014b). Psychology data from the "BAFACALO project: The Brazilian Intelligence Battery based on two state-of-the-art models - Carroll's Model and the CHC model". Journal of Open Psychology Data, 2(1), p.e6. doi: 10.5334/jopd.af

Golino, H. F., \& Gomes, C. M. A. (2014c). Visualizing random forest's prediction results. Psychology, 5, 2084-2098. doi: 10.4236/psych.2014.519211

Golino, H. F., Gomes, C. M. A., \& Andrade, D. (2014). Predicting academic achievement of high-school students using machine learning. Psychology, 5, 2046-2057. doi: 10.4236/psych.2014.518207

Golino, H. F., \& Gomes, C. M. A. (2015). Investigando estágios de desenvolvimento do raciocínio indutivo usando a análise fatorial confimatória, o Modelo Logístico Simples de Rasch e o modelo de teste logístico linear (Rasch Estendido). In H. F. Golino, C. M. Gomes, A. Amantes, \& G. Coelho, Psicometria Contemporânea: Compreendendo os Modelos Rasch (pp. 283-331). São Paulo: Casa do Psicólogo/Pearson.

Golino, H. F., \& Gomes, C. M. A. (2016). Random forest as an imputation method for education and psychology research: its impact on item fit and difficulty of the Rasch model. International Journal of Research \& Method in Education, 39(4), 401-421. doi: 10.1080/1743727X.2016.1168798

Golino, H. F. \& Gomes, C. M. A. (2019) TDRI: Teste de Desenvolvimento do Raciocínio Indutivo. São Paulo: Hogrefe.

Golino, H. F., Gomes, C. M. A., Amantes, A., \& Coelho, G. (2015). Psicometria contemporânea: compreendendo os Modelos Rasch (1 $1^{\text {ath }}$ ed., p. 416). São Paulo: Casa do Psicólogo. 
Golino, H. F., Gomes. C. M. A., Commons, M. L., \& Miller, P. M. (2014). The construction and validation of a developmental test for stage identification: Two exploratory studies. Behavioral Development Bulletin, 19(3), 37-54. doi: 10.1037/h0100589

Gomes, C. M. A. (2005). Uma análise dos fatores cognitivos mensurados pelo Exame Nacional do Ensino Médio (ENEM). Tese de Doutorado. Programa de Pós-Graduação em Educação da Universidade Federal de Minas Gerais. http://hdl.handle.net/1843/FAEC-85RJNN

Gomes, C. M. A. (2007a). Apostando no desenvolvimento da inteligência; em busca de um novo currículo educacional para o desenvolvimento do pensamento humano. Rio de Janeiro: Lamparina.

Gomes, C.M.A. (2007b). Softwares educacionais podem ser instrumentos psicológicos. Psicologia Escolar e Educacional, 11(2), 391-401. http://pepsic.bvsalud.org/scielo.php?script=sci_arttext\&pid=S1413$85572007000200016 \& \operatorname{lng}=$ pt\&tlng=pt.

Gomes, C. M. A. (2010a). Avaliando a avaliação escolar: notas escolares e inteligência fluida. Psicologia em Estudo, 15(4), 841-849. doi: 10.1590/S1413-73722010000400020

Gomes, C. M. A. (2010b). Estrutura fatorial da Bateria de Fatores Cognitivos de Alta-Ordem (BaFaCalo). Avaliação Psicológica, 9(3), 449-459.

http://pepsic.bvsalud.org/scielo.php?script=sci_arttext\&pid=S167704712010000300011\&lng=pt.

Gomes, C. M. A. (2010c). Perfis de estudantes e a relação entre abordagens de aprendizagem e rendimento Escolar. Psico (PUCRS. Online), 41(4), 503-509. http://revistaseletronicas.pucrs.br/ojs/index.php/revistapsico/article/view/6336

Gomes, C. M. A. (2011a). Abordagem profunda e abordagem superficial à aprendizagem: diferentes perspectivas do rendimento escolar. Psicologia: Reflexão e Crítica, 24(3), 438-447. doi: 10.1590/S0102-79722011000300004

Gomes, C. M. A. (2011b). Validade do conjunto de testes da habilidade de memória de curto-prazo (CTMC). Estudos de Psicologia (Natal), 16(3), 235-242. doi:10.1590/S1413-294X2011000300005

Gomes, C. M. A. (2012a). A estrutura fatorial do inventário de características da personalidade. Estudos de Psicologia (Campinas), 29(2), 209-220. doi:10.1590/S0103166X2012000200007

Gomes, C. M. A. (2012b). Validade de construto do conjunto de testes de inteligência cristalizada (CTIC) da bateria de fatores cognitivos de alta-ordem (BaFaCAlO). Gerais : Revista Interinstitucional de Psicologia, 5(2), 294-316. http://pepsic.bvsalud.org/scielo.php?script=sci_arttext\&pid=S1983$82202012000200009 \& \operatorname{lng}=$ pt\&tlng=pt. 
Gomes, C. M. A. (2013). A construção de uma medida em abordagens de aprendizagem. Psico (PUCRS. Online), 44(2), 193-203. http://revistaseletronicas.pucrs.br/ojs/index.php/revistapsico/article/view/11371

Gomes, C. M. A. (2020a). Análises estatísticas para estudos de intervenção. In M. MansurAlves \& J. B. Lopes-Silva, Intervenção cognitiva: dos conceitos às práticas baseadas em evidências para diferentes aplicações (pp. 93-107). Belo Horizonte: T.Ser.

Gomes, C. M. A. (2020b). Programa de Enriquecimento Instrumental: evidências de eficácia para intervenção cognitiva. In M. Mansur-Alves \& J. B. Lopes-Silva, Intervenção cognitiva: dos conceitos às práticas baseadas em evidências para diferentes aplicações (pp. 621-639). Belo Horizonte: T.Ser.

Gomes, C. M. A., \& Almeida, L. S. (2017). Advocating the broad use of the decision tree method in education. Practical Assessment, Research \& Evaluation, 22(10), 1-10. https://pareonline.net/getvn.asp?v=22\&n=10

Gomes, C. M. A., Almeida, L. S., \& Núñez, J. C. (2017). Rationale and applicability of exploratory structural equation modeling (ESEM) in psychoeducational contexts. Psicothema, 29(3), 396-401. doi: 10.7334/psicothema2016.369

Gomes, C.M.A., Amantes, A., \& Jelihovschi, E.G. (2020). Applying the regression tree method to predict students' science achievement. Trends in Psychology, 28, 99-117. doi: 10.9788/s43076-019-00002-5

Gomes, C. M. A., Araujo, J., Nascimento, E., \& Jelihovisch, E. (2018). Routine Psychological Testing of the Individual Is Not Valid. Psychological Reports, 122(4), 1576-1593. doi: 10.1177/0033294118785636

Gomes, C. M. A., Araujo, J., \& Jelihovschi, E. G. (2020). Approaches to learning in the nonacademic context: construct validity of Learning Approaches Test in Video Game (LAT-Video Game). International Journal of Development Research, 10(11), 4184241849. doi: 10.37118/ijdr.20350.11.2020

Gomes, C. M. A., \& Borges, O. N. (2007). Validação do modelo de inteligência de Carroll em uma amostra brasileira. Avaliação Psicológica, 6(2), 167-179. http://pepsic.bvsalud.org/scielo.php?script=sci_arttext\&pid=S167704712007000200007\&lng=en\&tlng=pt.

Gomes, C. M. A., \& Borges, O. N. (2008a). Avaliação da validade e fidedignidade do instrumento crenças de estudantes sobre ensino-aprendizagem (CrEA). Ciências \& Cognição (UFRJ), 13(3), 37-50.

http://www.cienciasecognicao.org/revista/index.php/cec/article/view/60

Gomes, C. M. A., \& Borges, O. (2008b). Limite da validade de um instrumento de avaliação docente. Avaliação Psicológica, 7(3), 391-401. http://pepsic.bvsalud.org/scielo.php?script=sci_arttext\&pid=S167704712008000300011\&lng=pt\&tlng=pt. 
Gomes, C. M. A., \& Borges, O. (2008c). Qualidades psicométricas de um conjunto de 45 testes cognitivos. Fractal: Revista de Psicologia, 20(1), 195-207. doi:10.1590/S198402922008000100019

Gomes, C. M. A. \& Borges, O. N. (2009a). O ENEM é uma avaliação educacional construtivista? Um estudo de validade de construto. Estudos em Avaliação Educacional, 20(42), 73-88. doi: 10.18222/eae204220092060

Gomes, C. M. A.s, \& Borges, O. N. (2009b). Propriedades psicométricas do conjunto de testes da habilidade visuo espacial. PsicoUSF, 14(1), 19-34. http://pepsic.bvsalud.org/scielo.php?script=sci_arttext\&pid=S1413$82712009000100004 \& \operatorname{lng}=$ pt\&tlng=pt.

Gomes, C. M. A., \& Borges, O. (2009c). Qualidades psicométricas do conjunto de testes de inteligência fluida. Avaliação Psicológica, 8(1), 17-32. http://pepsic.bvsalud.org/scielo.php?script=sci_arttext\&pid=S1677$04712009000100003 \& \operatorname{lng}=$ pt\&tlng=pt.

Gomes, C. M. A., de Araújo, J., Ferreira, M. G., \& Golino, H. F. (2014). The validity of the Cattel-Horn-Carroll model on the intraindividual approach. Behavioral Development Bulletin, 19(4), 22-30. doi: 10.1037/h0101078

Gomes, C. M. A., Fleith, D. S., Marinho-Araujo, C. M., \& Rabelo, M. L. (2020). Predictors of students' mathematics achievement in secondary education. Psicologia: Teoria e Pesquisa, 36, e3638. doi:10.1590/0102.3772e3638

Gomes, C. M. A., \& Gjikuria, J. (2017). Comparing the ESEM and CFA approaches to analyze the Big Five factors. Avaliação Psicológica, 16(3), 261-267. doi:10.15689/ap.2017.1603.12118

Gomes, C. M. A., \& Gjikuria, E. (2018). Structural Validity of the School Aspirations Questionnaire (SAQ). Psicologia: Teoria e Pesquisa, 34, e3438. doi:10.1590/0102.3772e 3438

Gomes, C. M. A., \& Golino, H. F. (2012a). O que a inteligência prediz: diferenças individuais ou diferenças no desenvolvimento acadêmico? Psicologia: teoria e prática, 14(1), 126-139. http://pepsic.bvsalud.org/scielo.php?script=sci_arttext\&pid=S1516$36872012000100010 \& \operatorname{lng}=\mathrm{pt} \& \operatorname{lng}=\mathrm{pt}$.

Gomes, C. M. A., \& Golino, H. F. (2012b). Relações hierárquicas entre os traços amplos do Big Five. Psicologia: Reflexão e Crítica, 25(3), 445-456. doi:10.1590/S010279722012000300004

Gomes, C. M. A., \& Golino, H. F. (2012c). Validade incremental da Escala de Abordagens de Aprendizagem (EABAP). Psicologia: Reflexão e Crítica, 25(4), 400-410. doi:10.1590/S0102-79722012000400001 
Gomes, C. M. A., \& Golino, H. F. (2014). Self-reports on students' learning processes are academic metacognitive knowledge. Psicologia: Reflexão e Crítica, 27(3), 472-480. doi: $10.1590 / 1678-7153.201427307$

Gomes, C. M. A., \& Golino, H. (2015). Factor retention in the intra-individual approach: Proposition of a triangulation strategy. Avaliação Psicológica, 14(2), 273-279. doi: 10.15689/ap.2015.1402.12

Gomes, C. M. A., Golino, H. F., \& Costa, B. C. G. (2013). Dynamic system approach in psychology: proposition and application in the study of emotion, appraisal and cognitive achievement. Problems of Psychology in the 21st Century, 6, 15-28. http://www.journals.indexcopernicus.com/abstracted.php?level=5\&icid=1059487

Gomes, C. M. A., Golino, H. F., \& Menezes, I. G. (2014). Predicting School Achievement Rather than Intelligence: Does Metacognition Matter? Psychology, 5, 1095-1110. doi: 10.4236/psych.2014.59122

Gomes, C. M. A., Golino, H. F., \& Peres, A. J. S. (2016). Investigando a validade estrutural das competências do ENEM: quatro domínios correlacionados ou um modelo bifatorial. Boletim na Medida (INEP-Ministério da Educação), 5(10), 33-30. http://portal.inep.gov.br/documents/186968/494037/BOLETIM+NA+MEDIDA++N\%C2\%BA+10/4b8e3d73-d95d-4815-866c-ac2298dff0bd?version=1.1

Gomes, C. M. A. Golino, H. F., \& Peres, A. J. S. (2018). Análise da fidedignidade composta dos escores do enem por meio da análise fatorial de itens. European Journal of Education Studies, 5(8), 331-344. doi:10.5281/zenodo.2527904

Gomes, C. M. A., Golino, H. F., \& Peres, A. J. S. (2020). Fidedignidade dos escores do Exame Nacional do Ensino Médio (Enem). Psico (RS), 54(2), 1-10. doi: 10.15448/19808623.2020.2.31145.

Gomes, C. M. A., Golino, H. F., Pinheiro, C. A. R., Miranda, G. R., \& Soares, J. M. T. (2011). Validação da Escala de Abordagens de Aprendizagem (EABAP) em uma amostra Brasileira. Psicologia: Reflexão e Crítica, 24(1), 19-27. doi: 10.1590/S010279722011000100004

Gomes, C. M. A., Golino, H. F., Santos, M. T., \& Ferreira, M. G. (2014). Formal-Logic Development Program: Effects on Fluid Intelligence and on Inductive Reasoning Stages. British Journal of Education, Society \& Behavioural Science, 4(9), 1234-1248. http://www.sciencedomain.org/review-history.php?iid=488\&id=21\&aid=4724

Gomes, C. M. A., \& Jelihovisch, E. (2016). Proposing a new approach and a rigorous cut-off value for identifying precognition. Measurement, 93, 117-125. doi: 10.1016/j.measurement.2016.06.066

Gomes, C. M. A., \& Jelihovschi, E. (2019). Presenting the regression tree method and its application in a large-scale educational dataset. International Journal of Research \& Method in Education. doi: 10.1080/1743727X.2019.1654992 
Gomes, C. M. A., Lemos, G. C., \& Jelihovschi, E. G. (2020). Comparing the predictive power of the CART and CTREE algorithms. Avaliação Psicológica, 19(1), 87-96. doi: 10.15689/ap.2020.1901.17737.10

Gomes, C. M. A, \& Linhares. (2018). Investigação da validade de conteúdo do TAPPensamento. Pôster. I Encontro Anual da Rede Nacional de Ciência para Educação (CPE). doi: 10.13140/RG.2.2.31110.40006

Gomes, C. M. A., Linhares, I. S., Jelihovschi, E. G., \& Rodrigues, M. N. S. (2021). Introducing rationality and contente validity of SLAT-Thinking. International Journal of Development Research, 11(1), 43264-43272, doi: 10.37118/ijdr.20586.01.2021

Gomes, C. M. A., \& Marques, E. L. L. (2016). Evidências de validade dos estilos de pensamento executivo, legislativo e judiciário. Avaliação Psicológica, 15(3), 327-336. doi: 10.15689/ap.2016.1503.05

Gomes, C. M. A., Marques, E. L. L., \& Golino, H. F. (2014). Validade Incremental dos Estilos Legislativo, Executivo e Judiciário em Relação ao Rendimento Escolar. Revista E-Psi, 2, 31-46. https://revistaepsi.com/artigo/2013-2014-ano3-volume2-artigo3/

Gomes, C. M. A., Nascimento, E., \& Peres, A. J. S. (2019). Investigating causal relations in personality by combining path analysis and Search algoritms. Poster. 3rd World Conference on Personality, World Association for Personality Psychology (WAPP), Hanoi, Vietnam.

Gomes, C. M. A., \& Nascimento, D. F. (2021a). A medida da habilidade de fluência do modelo CHC: apresentando o Teste de Fluência Ideativa 2 da Bateria de Fatores Cognitivos de Alta-Ordem (BAFACALO). Preprint. doi:

10.13140/RG.2.2.35726.28481/1.

https://www.researchgate.net/publication/349225691_A_Medida_da_Habilidade_de_Fl uencia_do_Modelo_CHC_apresentando_o_Teste_de_Fluencia_Ideativa_2_da_Bateria_ de_Fatores_Cognitivos_de_Alta-Ordem_BAFACALO

Gomes, C. M. A., \& Nascimento, D. F. (2021b). Acesso aberto ao Teste de Fluência Figural da Bateria de Fatores Cognitivos de Alta-Ordem (BAFACALO) como medida da habilidade ampla de fluência do modelo CHC de inteligência. Preprint. doi: 10.13140/RG.2.2.15593.62564/1. https://www.researchgate.net/publication/349225605_Acesso_Aberto_ao_Teste_de_Flu encia_Figural_da_Bateria_de_Fatores_Cognitivos_de_AltaOrdem_BAFACALO_como_Medida_da_Habilidade_Ampla_de_Fluencia_do_Modelo_ CHC_de_Inteligencia

Gomes, C. M. A., \& Nascimento, D. F. (2021c). Acesso aberto e gratuito ao Teste de Fluência Ideativa 1 da BAFACALO. Preprint. doi: 10.13140/RG.2.2.24821.09442/3. https://www.researchgate.net/publication/349225867_Acesso_Aberto_e_Gratuito_ao_T este_de_Fluencia_Ideativa_1_da_BAFACALO 
Gomes, C. M. A., \& Nascimento, D. F. (2021d). Acesso aberto e gratuito ao Conjunto de Testes de Inteligência Fluida: Teste de Raciocínio Geral da Bateria de Fatores Cognitivos de Alta-Ordem (BAFACALO). Preprint. doi:

10.13140/RG.2.2.30509.61921/1.

https://www.researchgate.net/publication/349213277_Acesso_Aberto_e_Gratuito_ao_C onjunto_de_Testes_de_Inteligencia_Fluida_Teste_de_Raciocinio_Geral_da_Bateria_de _Fatores_Cognitivos_de_Alta-Ordem_BAFACALO

Gomes, C. M. A., \& Nascimento, D. F. (2021e). Apresentando o Teste de Flexibilidade de Fechamento da BAFACALO. Preprint. doi: 10.13140/RG.2.2.31920.28164. https://www.researchgate.net/publication/349537118_Apresentando_o_Teste_de_Flexib ilidade_de_Fechamento_da_BAFACALO

Gomes, C. M. A., \& Nascimento, D. F. (2021f). Disponibilizando de forma gratuita e aberta o Teste de Memória Associativa 1 da Bateria de Fatores Cognitivos de Alta-Ordem (BAFACALO). Preprint. doi: 10.13140/RG.2.2.29964.03201/1. https://www.researchgate.net/publication/349409427_Disponibilizando_de_Forma_Grat uita_e_Aberta_o_Teste_de_Memoria_Associativa_1_da_Bateria_de_Fatores_Cognitivo s_de_Alta-Ordem_BAFACALO

Gomes, C. M. A., \& Nascimento, D. F. (2021g). Disponibilizando de forma gratuita e aberta o Teste de Velocidade Numérica da BAFACALO. Preprint. doi:

10.13140/RG.2.2.24114.94407/1. https://www.researchgate.net/publication/349670492_Disponibilizando_de_Forma_Grat uita_e_Aberta_o_Teste_de_Velocidade_Numerica_da_BAFACALO

Gomes, C. M. A., \& Nascimento, D. F. (2021h). Medidas de inteligência cristalizada: disponibilizando o Teste de Compreensão Verbal 2 da Bateria de Fatores Cognitivos de Alta-Ordem (BAFACALO). Preprint. doi: 10.13140/RG.2.2.36085.09447/1. https://www.researchgate.net/publication/349349602_Medidas_de_Inteligencia_Cristali zada_Disponibilizando_o_Teste_de_Compreensao_Verbal_2_da_Bateria_de_Fatores_C ognitivos_de_Alta-Ordem_BAFACALO

Gomes, C. M. A., \& Nascimento, D. F. (2021i). Medindo a habilidade de rapidez cognitiva do modelo CHC: apresentando o Teste de Velocidade Perceptiva 1 da BAFACALO. Preprint. doi: 10.13140/RG.2.2.28564.83848/1. https://www.researchgate.net/publication/349537123_Medindo_a_Habilidade_de_Rapid ez_Cognitiva_do_Modelo_CHC_Apresentando_o_Teste_de_Velocidade_Perceptiva_1_ da_BAFACALO

Gomes, C. M. A., \& Nascimento, D. F. (2021j). Presenting SLAT-Thinking Second Version and its content validity. International Journal of Development Research, 11(3), 4559045596. doi: 10.37118/ijdr.21368.03.2021

Gomes, C. M. A., \& Nascimento, D. F. (2021k). Projeto de acesso aberto e gratuito aos testes do LAICO: Teste de Raciocínio Lógico da Bateria de Fatores Cognitivos de AltaOrdem (BAFACALO). Preprint. doi: 10.13140/RG.2.2.25476.45445/1. 
https://www.researchgate.net/publication/349213384_Projeto_de_Acesso_Aberto_e_Gr atuito_aos_Testes_do_LAICO_Teste_de_Raciocinio_Logico_da_Bateria_de_Fatores_C ognitivos_de_Alta-Ordem_BAFACALO

Gomes, C. M. A., \& Nascimento, D. F. (20211). Projeto de acesso aberto e gratuito à Bateria de Fatores Cognitivos de Alta-Ordem (BAFACALO): o Teste de Compreensão Verbal 1 do Conjunto de Testes de Inteligência Cristalizada. Preprint. doi:

10.13140/RG.2.2.22663.32165/1.

https://www.researchgate.net/publication/349349694_Projeto_de_Acesso_Aberto_e_Gr atuito_a_Bateria_de_Fatores_Cognitivos_de_Alta-

Ordem_BAFACALO_o_Teste_de_Compreensao_Verbal_1_do_Conjunto_de_Testes_d e_Inteligencia_Cristalizada

Gomes, C. M. A., \& Nascimento, D. F. (2021m). Projeto de acesso aos testes de inteligência da BAFACALO: Teste de Compreensão Verbal 3. Preprint. doi:

10.13140/RG.2.2.10499.84001/2.

https://www.researchgate.net/publication/349349798_Projeto_de_Acesso_aos_Testes_d e_Inteligencia_da_BAFACALO_Teste_de_Compreensao_Verbal_3

Gomes, C. M. A., \& Nascimento, D. F. (2021n). Projeto de acesso da BAFACALO: Teste de Memória Associativa 2. Preprint. doi: 10.13140/RG.2.2.23253.14565/1.

https://www.researchgate.net/publication/349409430_Projeto_de_Acesso_da_BAFACA LO_Teste_de_Memoria_Associativa_2

Gomes, C. M. A., \& Nascimento, D. F. (2021o). Teste de Memória Visual da Bateria de Fatores Cognitivos de Alta-Ordem (BAFACALO). Preprint. doi:

10.13140/RG.2.2.33319.47529.

https://www.researchgate.net/publication/349409522_Teste_de_Memoria_Visual_da_B ateria_de_Fatores_Cognitivos_de_Alta-Ordem_BAFACALO

Gomes, C. M. A., Nascimento, D. F., \& Araujo, J. (2021a). Medindo a inteligência fluida: o Teste de Indução da Bateria de Fatores Cognitivos de Alta-Ordem (BAFACALO).

Preprint. doi: 10.13140/RG.2.2.17087.84641/3. https://www.researchgate.net/publication/349213375_Medindo_a_Inteligencia_Fluida_ o_Teste_de_Inducao_da_Bateria_de_Fatores_Cognitivos_de_AltaOrdem_BAFACALO

Gomes, C. M. A., \& Nascimento, D. F., \& Araujo, J. (2021b). Acesso aberto ao Teste de Dobraduras (VZ) da BAFACALO. Preprint. doi: 10.13140/RG.2.2.21853.95201/2. https://www.researchgate.net/publication/349536932_Acesso_Aberto_ao_Teste_de_Do braduras_VZ_da_BAFACALO

Gomes, C. M. A., \& Nascimento, D. F., \& Araujo, J. (2021c). Projeto de testes gratuitos e abertos do LAICO: Teste de Velocidade Perceptiva 3 da BAFACALO. Preprint. doi: 10.13140/RG.2.2.36278.42563/2. https://www.researchgate.net/publication/349670486_Projeto_de_Testes_Gratuitos_e_A bertos_do_LAICO_Teste_de_Velocidade_Perceptiva_3 da_BAFACALO 
Gomes, C. M. A., \& Nascimento, D. F., \& Araujo, J. (2021d). Teste de Velocidade Perceptiva 2 da Bateria de Fatores Cognitivos de Alta-Ordem (BAFACALO): disponibilização aberta e gratuita aos testes de medida de rapidez cognitiva do LAICO. Preprint. doi: 10.13140/RG.2.2.29567.53928/1. https://www.researchgate.net/publication/349670396_Teste_de_Velocidade_Perceptiva _2_da_Bateria_de_Fatores_Cognitivos_de_AltaOrdem_BAFACALO_Disponibilizacao_Aberta_e_Gratuita_aos_Testes_de_Medida_de _Rapidez_Cognitiva_do_LAICO

Gomes, C. M. A., \& Valentini, F. (2019). Time series in educational psychology: application in the study of cognitive achievement. European Journal of Education Studies, 6(8), 214-229. doi: 10.5281/zenodo.3551953

Jelihovschi, E. G., \& Gomes, C. M. A. (2019). Proposing an achievement simulation methodology to allow the estimation of individual in clinical testing context. Revista Brasileira de Biometria, 37(4), 1-10. doi: 10.28951/rbb.v37i4.423

Laros, J. A., Valentini, F., Gomes, C. M. A., \& Andrade, J. M. (2014). Modelos de inteligência. In A. G. Seabra, J. A. Laros, E. C. Macedo \& N. Abreu (Eds.), Inteligência e funções executivas:Avanços e desafios para a avaliação neuropsicológica (pp. 17-38). São Paulo: Editora Memnon

Martins, A. A., Gomes, C. M. A., Alves, A. F., Almeida, L. S. (2018). The structure of intelligence in childhood: age and socio-familiar impacto $\mathrm{n}$ cognitive differentiation. Psychological Reports, 121(1), 79-92. doi: 10.1177/0033294117723019

Matos, D. A. S., Brown, G. T. L., \& Gomes, C. M. A. (2019). Bifactor invariance analysis of student conceptions of assessment inventory. Psico-USF, 24(4), 737-750. doi: $10.1590 / 1413-82712019240411$

Mecca, T. P., Dias, N. M, Reppold, C. R, Muniz, M., Gomes, C. M. A., Fioravanti-Bastos, A. C. M., Yates, D. B., Carreiro, L. R. R., \& Macedo, E. C. (2015). Funcionamento adaptativo: panorama nacional e avaliação com o adaptive behavior assessment system. Psicologia: Teoria e Prática, 17(2), 107-122, doi:0.15348/19806906/psicologia.v17n2p107-122

Monteiro, S., Almeida, L. S., Gomes, C. M. A., \& Sinval, J. (2020). Employability profiles of higher education graduates: a person-oriented approach. Studies in Higher Education. doi: 10.1080/03075079.2020.1761785

Moura, M. D. G., Gomes, C. M. A., Blanc, S. L., Mesquita, R. A., \& Ferreira, E. F. (2014). Development of questionnaire on dentists? knowledge of HIV/AIDS. Arquivos em Odontologia (UFMG. Online), 50(1), 6-12, 2014. doi:10.7308/aodontol/2014.50.1.01

Muniz, M., Gomes, C. M. A., \& Pasian, S. R. (2016). Factor structure of Raven's Coloured Progressive Matrices. Psico-USF, 21(2), 259-272. doi: 10.1590/1413-82712016210204 
Pazeto, T. C. B., Dias, N. M., Gomes, C. M. A., \& Seabra, A. G. (2019). Prediction of arithmetic competence: role of cognitive abilities, socioeconomic variables and the perception of the teacher in early childhood education. Estudos de Psicologia, 24(3), 225-236. doi: 10.22491/1678-4669.20190024

Pazeto, T. C. B., Dias, N. M., Gomes, C. M. A., \& Seabra, A. G. (2020). Prediction of reading and writing in elementary education through early childhood education. Psicologia: Ciência e Profissão, 40, e205497, 1-14. doi: 10.1590/1982-3703003205497

Pereira, B. L. S., Golino, M. T. S., \& Gomes, C. M. A. (2019). Investigando os efeitos do programa de enriquecimento instrumental básico em um estudo de caso único. European Journal of Education Studies, 6(9). doi:10.46827/ejes.v0i0.2669

Pinheiro, C. A. R., Gomes, C. M. A., \& Braga, A. G. (2009). Construção e validação do Inventário dos Adjetivos de Personalidade - 50 (IAP-50) [Resumo]. In Anais do IV Congresso Brasileiro de Avaliação Psicológica e XIV Conferência Internacional de Avaliação Psicológica: Formas e Contextos (p. 182). Campinas, SP: Instituto Brasileiro de Avaliação Psicológica.

Pires, A. A. M., \& Gomes, C. M. A. (2017). Three mistaken procedures in the elaboration of school exams: explicitness and discussion. PONTE International Scientific Researches Journal, 73(3), 1-14. doi: 10.21506/j.ponte.2017.3.1

Pires, A. A. M., \& Gomes, C. M. A. (2018). Proposing a method to create metacognitive school exams. European Journal of Education Studies, 5(8), 119-142. doi:10.5281/zenodo. 2313538

Reppold, C. T., Gomes, C. M. A., Seabra, A. G., Muniz, M., Valentini, F., \& Laros, J.A. (2015). Contribuições da psicometria para os estudos em neuropsicologia cognitiva. Psicologia: teoria e prática, 17(2), 94-106. doi: 10.15348/19806906/psicologia.v17n2p94-106

Ricci, K., Gomes, C. M. A., Nico, M. A. N., Seabra, A. G. (2020). Programa de Enriquecimento Instrumental (PEI) Básico em crianças com TDAH e dislexia. Psicologia desde el Caribe, 37(3). http://rcientificas.uninorte.edu.co/index.php/psicologia/article/view/12397

Rodrigues, M. N. S., \& Gomes, C. M. A. (2020). Testing the hypothesis that the deep approach generates better academic performance. International Journal of Development Research, 10(12), 42925-42935. doi:10.37118/ijdr.20579.12.2020

Rosa, M. A. C., Gomes, C. M. A., Rocha, N. S., Kessler, F. H. P., Slavutzky, S. M. B., Ferreira, E. F., \& Pechansky, F. (2013). Dependence module of the MINI plus adapted for sugar dependence: psychometric properties. Psicologia: Reflexão e Crítica, 26(1), 77-86. doi:10.1590/S0102-79722013000100009 
Rosário, V. M., Gomes, C. M. A., \& Loureiro, C. M. V. (2019). Systematic review of attention testing in allegedly "untestable" populations. International Journal of Psychological Research and Reviews, 2(19), 1-21. doi: 10.28933/ijprr-2019-07-1905

Sampaio, R. T., Loureiro, C. M. V., \& Gomes, C. M. A. (2015). A Musicoterapia e o Transtorno do Espectro do Autismo: uma abordagem informada pelas neurociências para a prática clínica. Per Musi, 32, 137-170. doi:10.1590/permusi2015b3205

Silveira, M. S., \& Gomes, C. M. A. (2014). Avaliação do desenvolvimento experiencial de pacientes com prótese ocular: a focalização no atendimento clínico. Psicologia Clínica, 26(1), 181-196. http://pepsic.bvsalud.org/scielo.php?script=sci_arttext\&pid=S0103$56652014000100012 \& \operatorname{lng}=$ pt\&tlng=pt.

Silveira, M. B., Gomes, C. M. A., Golino, H. F., \& Dias, F. S. (2012). Construção do Teste de Habilidade Experiencial (THE): Evidências iniciais de validade e confiabilidade. Revista E-psi, 1, 77-96. https://revistaepsi.com/artigo/2012-ano2-volume1-artigo4/

Therneau, T. \& Atkinson, B. (2019). rpart: Recursive Partitioning and Regression Trees. R package version 4.1-15. https://CRAN.R-project.org/package=rpart

Valentini, F., Gomes, C. M. A., Muniz, M., Mecca, T. P., Laros, J. A., \& Andrade, J. M. (2015). Confiabilidade dos índices fatoriais da Wais-III adaptada para a população brasileira. Psicologia: teoria e prática, 17(2), 123-139. doi: 10.15348/19806906/psicologia.v17n2p123-139

\section{Appendix}

\section{The $\mathbf{R}$ code to simulate the trees}

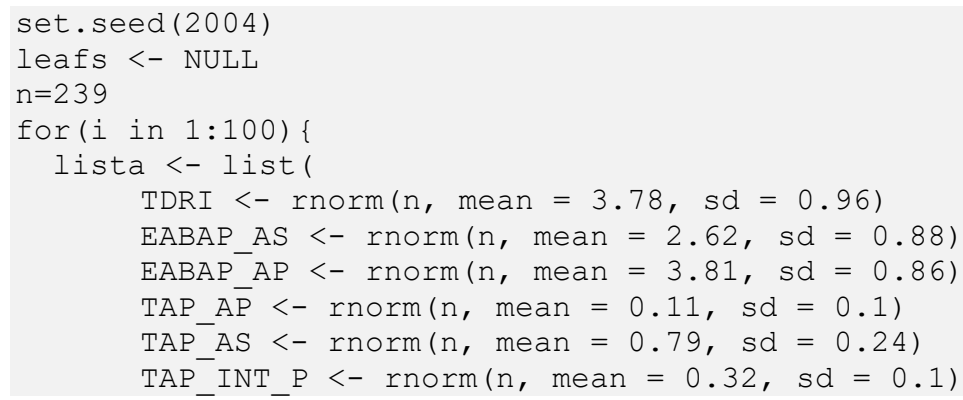




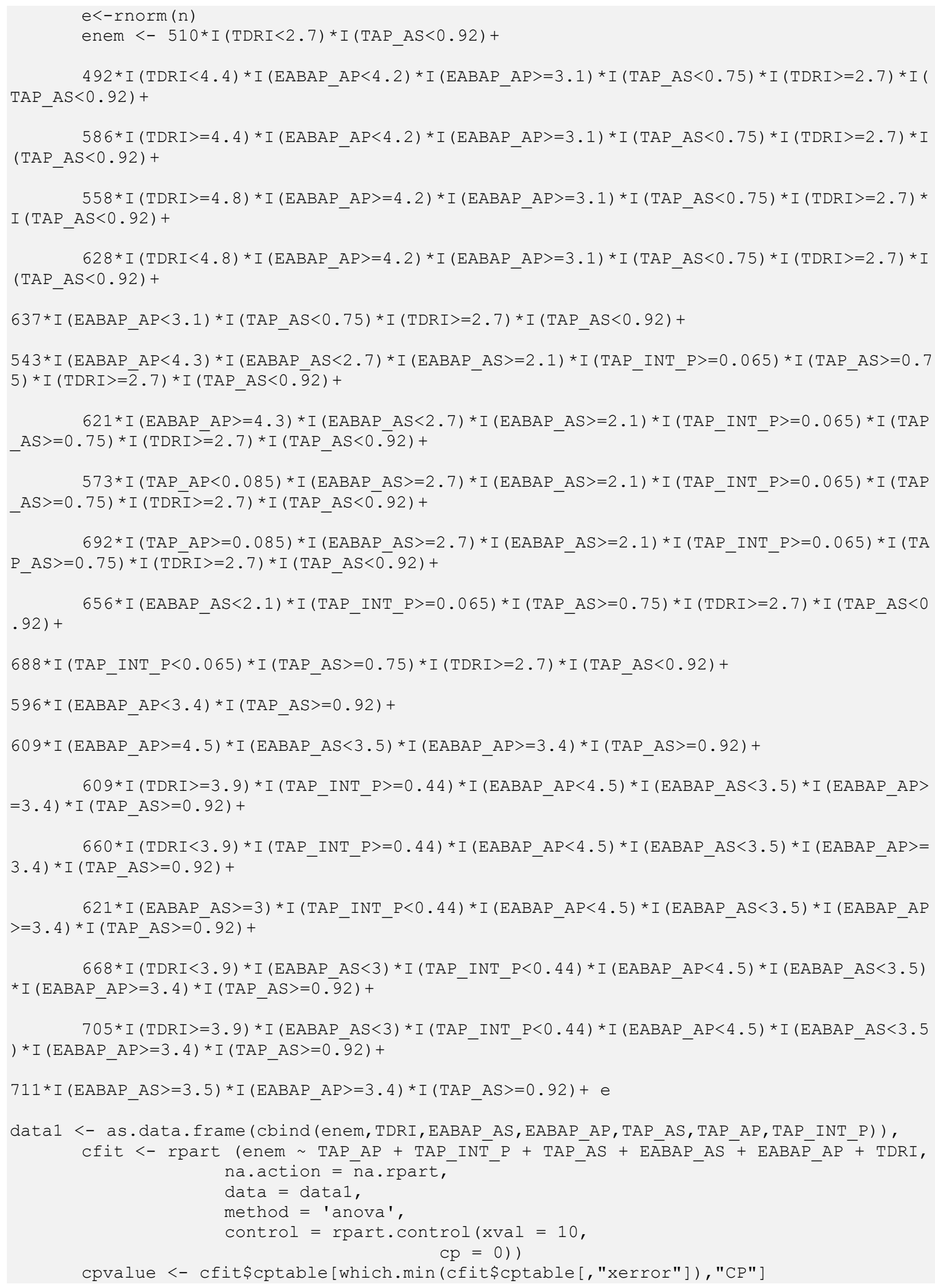




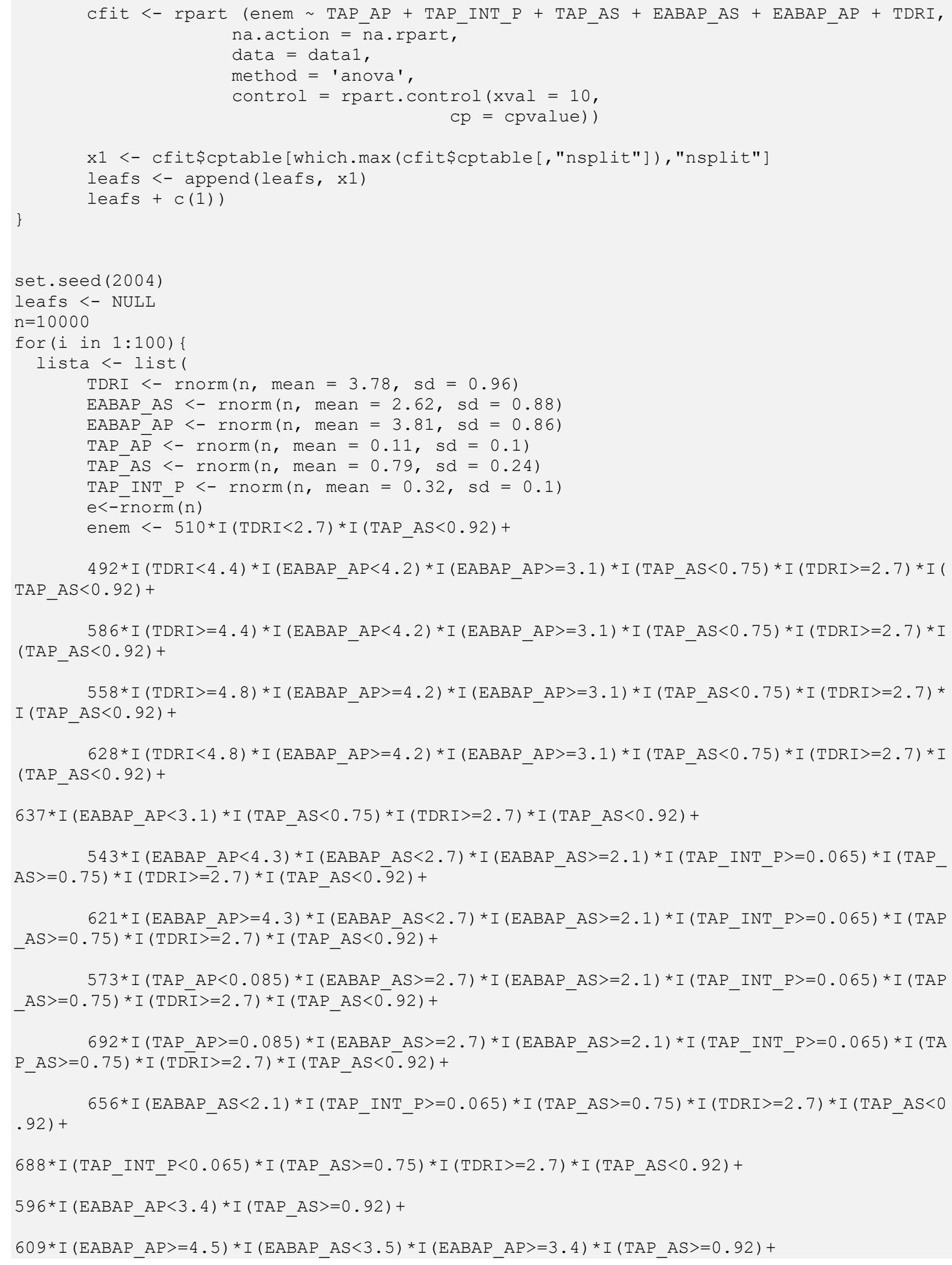




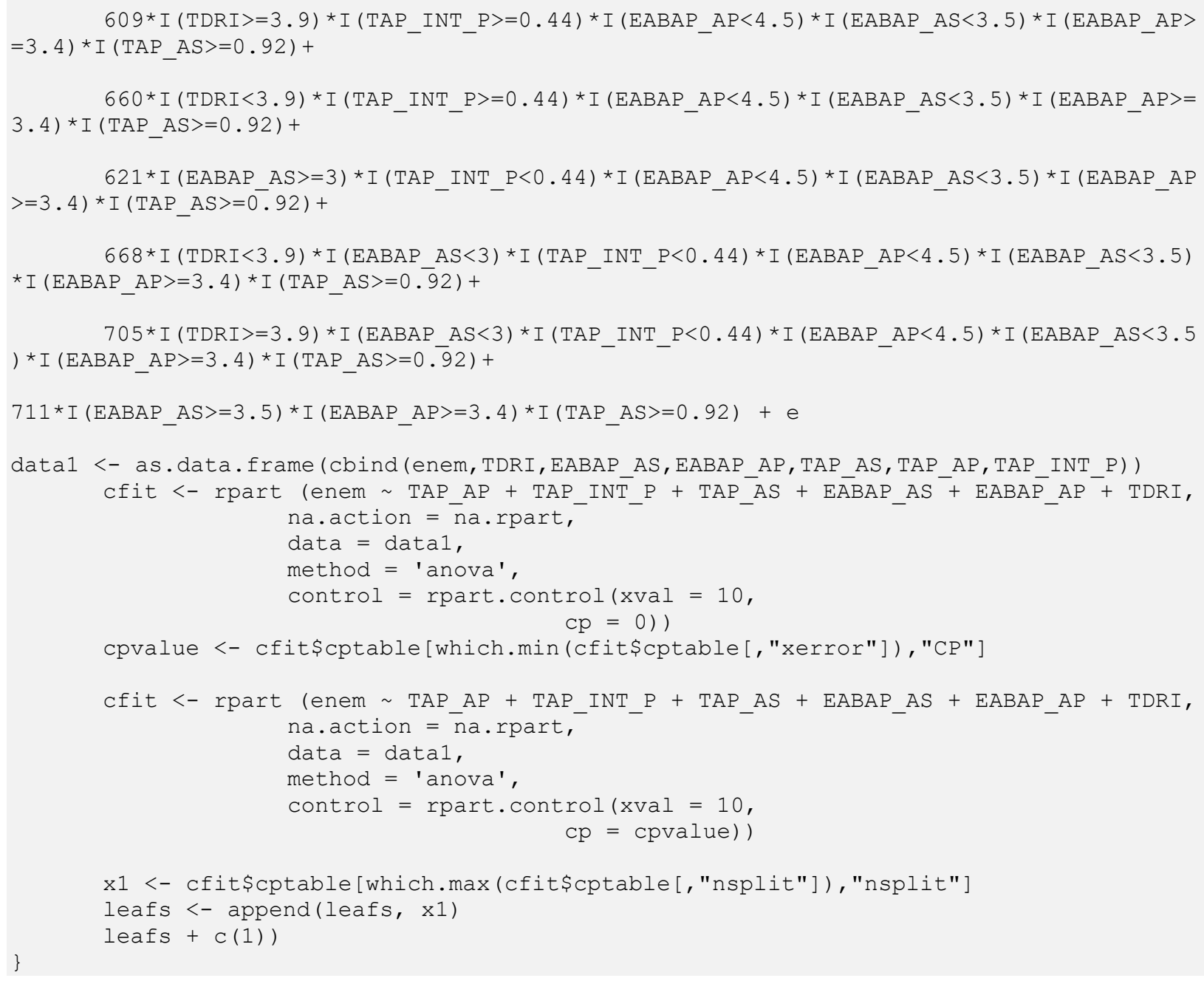

